\title{
Efficient high-order evaluation of scattering by periodic surfaces: deep gratings, high frequencies, and glancing incidences
}

\author{
Oscar P. Bruno ${ }^{1}$ and Michael C. Haslam ${ }^{2, *}$ \\ ${ }^{1}$ Applied and Computational Mathematics, Caltech, Pasadena, California 91125, USA \\ ${ }^{2}$ Department of Mathematics and Statistics, York University, Toronto, Ontario M3J 1P3, Canada \\ *Corresponding author: mchaslam@mathstat.yorku.ca
}

\author{
Received October 30, 2008; accepted December 10, 2008; \\ posted December 22, 2008 (Doc. ID 103378); published February 25, 2009
}

\begin{abstract}
We present a superalgebraically convergent integral equation algorithm for evaluation of TE and TM electromagnetic scattering by smooth perfectly conducting periodic surfaces $z=f(x)$. For grating-diffraction problems in the resonance regime (heights and periods up to a few wavelengths) the proposed algorithm produces solutions with full double-precision accuracy in single-processor computing times of the order of a few seconds. The algorithm can also produce, in reasonable computing times, highly accurate solutions for very challenging problems, such as (a) a problem of diffraction by a grating for which the peak-to-trough distance equals 40 times its period that, in turn, equals 20 times the wavelength; and (b) a high-frequency problem with very small incidence, up to $0.01^{\circ}$ from glancing. The algorithm is based on the concurrent use of Floquet and Chebyshev expansions together with certain integration weights that are computed accurately by means of an asymptotic expansion as the number of integration points tends to infinity. (C) 2009 Optical Society of America

OCIS codes: $050.1755,050.5745$.
\end{abstract}

\section{INTRODUCTION}

The problem of evaluating the scattering of waves by periodic surfaces is one of fundamental importance in science and engineering. A vast literature has thus developed in this area: an excellent survey of early approaches to this problem is given in the classical reference [1]; other reviews and contributions, spanning many decades, include [2-6] among many others. Significant progress has emerged from these efforts. Yet, an examination of the literature reveals that the manifold approaches to this problem share certain limitations: typically diminishing accuracies can be achieved as the surface height-toperiod ratios and/or frequencies are increased, and as grazing angles approach zero degrees. Beyond certain values of these parameters, no accuracies have been demonstrated by any of the existing methodologies.

In this paper we propose a numerical method that can treat a significantly larger range of scattering problems than have been previously treated. A case study is presented, for example, in which the depth of the scattering surface is 40 times its period and for which the period equals 20 times the wavelength (in particular, the period equals 800 times the wavelength); our solver produced the solution for this problem with full ten digits accuracy. The closest example we found in the literature is given in [3]: there a surface of depth equal to eight times its period is presented, for which the solution was produced with an accuracy of three digits. Other rather extreme cases are considered in the present text, including cases involving high frequencies and small grazing angles. For wavelengths in the resonance regime (where wavelengths are comparable to the period), further, the present approach can produce solutions with full double-precision accuracies in computing times of the order of a few seconds on contemporary single-processor personal computers.

Our method is based on use of integral equations [7], representation of the unknown surface densities in terms of a Floquet series, and superalgebraically accurate evaluation of all integrals involving singular kernels by means of appropriate use of Chebyshev expansions. In this contribution we confine our discussion to problems of scattering by perfectly conducting smooth periodic surfaces, but the approach can be extended to other cases, including nonperfectly conducting, penetrable surfaces, and nonsmooth geometries.

The aforementioned contribution [3] includes a survey of modern integral equation methods that provides an excellent frame of reference for our methods and results. The most robust mathematically sound approach considered in that reference is a certain collocation method (referred to as CC in [3]). The essentials of the CC method are related to the present approach. The present work differs from the CC and related methods of [3] in some fundamental ways, however: the CC method uses the kernel decomposition

$$
K\left(x, x^{\prime}\right)=-\frac{1}{2 \pi} \ln \left|x-x^{\prime}\right|+\tilde{K}\left(x, x^{\prime}\right),
$$

which contains the singular component $\widetilde{K}$ : the first derivative of this function is unbounded at $x=x^{\prime}$. Numerical integration schemes for integrands containing such singularities that do not explicitly account for the derivative singularities exhibit low-order convergence. The key ele- 
ments of the algorithm proposed in this text are, precisely, (1) use of a decomposition of the form

$$
K\left(x, x^{\prime}\right)=K_{1}\left(x, x^{\prime}\right) \ln \left|x-x^{\prime}\right|+K_{2}\left(x, x^{\prime}\right),
$$

(where $K_{1}$ and $K_{2}$ are smooth or even analytic functions of their arguments, provided the scattering surface is, respectively, smooth or analytic), together with (2) a methodology, based on Chebyshev representations and use of asymptotic expansions for evaluation of integration weights, for fast and highly accurate evaluation of integrals of products of the kernel and the unknown integral density. As a result of this approach we obtain a fast superalgebraically convergent algorithm: the errors in the solutions obtained by using $N$ unknowns and $M$ kernel weights tend to zero faster than $\mathcal{O}\left(N^{-m}\right)$ and $\mathcal{O}\left(M^{-m}\right)$ for all positive integers $m$. As discussed above, use of this algorithm allows for treatment of greatly challenging grating-diffraction problems with high accuracy and speed.

This paper is organized as follows: after introducing the relevant integral equations and discussing the extraction of the kernel singularity in Sections 2 and 3, in Sections 4 we present the main lines of our integral equation solver-which, as mentioned above, is based on concurrent use of Floquet and Chebyshev expansions; in particular, Subsection 4.C treats a somewhat subtle matter concerning fast and accurate evaluation of integration weights. In Section 5, finally, we present a variety of numerical results demonstrating the qualities of the overall proposed methodology.

\section{INTEGRAL EQUATIONS}

We consider the problem of evaluating the scattering of a time-harmonic incident plane wave of wavelength $\lambda$ $=2 \pi / k$,

$$
u_{\text {inc }}(\mathbf{r})=\exp [i(\alpha x-\beta z)]
$$

with

$$
\alpha=k \cos \theta, \quad \beta=k \sin \theta,
$$

by a perfectly conducting periodic surface $z=f(x)$, where $f: \mathrm{R} \rightarrow \mathrm{R}$ is a smooth $P$-periodic function, $f(x)=f(x+P)$; clearly $\theta>0$ denotes the incidence angle measured from the horizontal. The total field $u(\mathbf{r})$, which equals the sum $u_{i n c}(\mathbf{r})+u_{s}(\mathbf{r})$ of the incident field $u_{i n c}$ and the (radiating) scattered field $u_{s}$, satisfies the scalar Helmholtz equation

$$
\Delta u+k^{2} u=0, \quad \mathbf{r} \in \mathcal{S},
$$

where $\mathcal{S}=\left\{\mathbf{r}=(x, z) \in \mathbb{R}^{2}: z>f(x)\right\}$. The TE and TM boundary-value problems for the Helmholtz equation result as the respective boundary conditions $u=0$ and $\partial u / \partial \mathbf{n}=0$ are imposed at the interface $\partial \mathcal{S}=\{z=f(x)\}$, where $\mathbf{n}(\mathbf{r})$ denotes the upward normal unit vector to $\partial \mathcal{S}$.

As is known, the solutions of Eq. (4) for both TE and TM polarizations can be expressed in terms of singlelayer potentials of the form

$$
u_{s}(\mathbf{r})=\int_{\partial \mathcal{S}} \Phi\left(\mathbf{r}, \mathbf{r}^{\prime}\right) \mu\left(\mathbf{r}^{\prime}\right) \mathrm{d} s\left(\mathbf{r}^{\prime}\right), \quad \mathbf{r} \in \mathcal{S}
$$

where $\mu$ is an (unknown) surface density function and where $\Phi\left(\mathbf{r}, \mathbf{r}^{\prime}\right)=i H_{0}^{(1)}\left(i k\left|\mathbf{r}-\mathbf{r}^{\prime}\right|\right) / 4$ is the two-dimensional radiating free-space Green's function ([8], Eq. 3.60). The surface densities $\mu^{+}$and $\mu^{-}$associated with TE and TM polarizations are solutions of the integral equations

$$
\frac{\partial u_{\mathrm{inc}}(\mathbf{r})}{\partial \mathbf{n}(\mathbf{r})}=\frac{1}{2} \mu^{ \pm}(\mathbf{r}) \pm \int_{\partial \mathcal{S}} \frac{\partial \Phi\left(\mathbf{r}, \mathbf{r}^{\prime}\right)}{\partial \mathbf{n}(\mathbf{r})} \mu^{ \pm}\left(\mathbf{r}^{\prime}\right) \mathrm{d} s\left(\mathbf{r}^{\prime}\right), \quad \mathbf{r} \in \partial \mathcal{S}
$$

where the plus sign is used for TE polarization, and the minus sign is used in the TM case [5]. The TM equation results as the normal derivative of Eq. (5) is computed in the limit as $\mathbf{r}$ approaches the interface $\partial \mathcal{S}$ from above. In the case of TE polarization the derivation of Eq. (6) involves Green's formula, and we have $\mu^{+}=-\partial u / \partial n$. No such physical interpretation exists for the TM polarization density $\mu^{-}$.

As is customary, we re-express the single- and doublelayer integrals in Eqs. (5) and (6), whose integration domain is the infinite surface $\partial \mathcal{S}$, as an integral over a single period $\mathcal{P}$ of $\partial \mathcal{S}$. This reduction can indeed be effected in view of the quasi-periodicity conditions $u(\mathbf{r}+\hat{\mathbf{x}} P)$ $=\exp (i \alpha P) u(\mathbf{r})$ and $\mu^{ \pm}(\mathbf{r}+\hat{\mathbf{x}} P)=\exp (i \alpha P) \mu^{ \pm}(\mathbf{r}) \quad[\hat{\mathbf{x}}=(1,0)]$ satisfied by the solutions $u$ and $\mu^{ \pm}$. For the scattered field Eq. (5), for example, we obtain an expression of the form

$$
u_{s}(\mathbf{r})=\int_{\mathcal{P}} G\left(\mathbf{r}, \mathbf{r}^{\prime}\right) \mu\left(\mathbf{r}^{\prime}\right) d s\left(\mathbf{r}^{\prime}\right)
$$

where $G$ is the free-space periodic Green's function; see, e.g., [1]. Denoting $\alpha_{n}=\alpha+2 n \pi / P$ and $\beta_{n}=\sqrt{k^{2}-\alpha_{n}^{2}}$ for $k$ $\geqslant \alpha_{n}, \beta_{n}=i \sqrt{\alpha_{n}^{2}-k^{2}}$ for $k<\alpha_{n}$, we have $[1,9]$

$$
G\left(\mathbf{r}, \mathbf{r}^{\prime}\right)=\frac{i}{2 P} \sum_{n=-\infty}^{\infty} \frac{e^{i \alpha_{n}\left(x-x^{\prime}\right)+i \beta_{n}\left|z-z^{\prime}\right|}}{\beta_{n}} .
$$

The spectral series representation, Eq. (8), converges uniformly in compact subsets of $\left\{z \neq z^{\prime}\right\}$, but it cannot be termwise differentiated with respect to $z$ or $z^{\prime}$ at $z=z^{\prime}$ [9]. The representation, Eq. (8), is suitable for the numerical evaluation of the periodic Green's function for adequately large values of $k\left|z-z^{\prime}\right|$; in Appendix A we present algorithms for the efficient evaluation of certain analytic functions $K_{1}$ and $K_{2}$ in an expression of the form, Eq. (2), for the normal derivative of the periodic Green's function $G$.

The line of reasoning used to reduce the integral in Eq. (5) to an integral, Eq. (7), over a single period can be applied to the integral equation (6): denoting

$$
\phi(x)=\left(1+\left[f^{\prime}(x)\right]^{2}\right)^{1 / 2} \mu(\mathbf{s}(x)),
$$

where $\mathbf{s}(x)=(x, f(x))$ is a surface position vector, the integral equations (6) can be expressed in the form 


$$
\psi(x)=\frac{1}{2} \phi(x) \pm \int_{x-P / 2}^{x+P / 2} K\left(x, x^{\prime}\right) \phi\left(x^{\prime}\right) \mathrm{d} x^{\prime} .
$$

The kernel and source function in the second-kind Fredholm equation (10) are given by

$$
\begin{aligned}
K\left(x, x^{\prime}\right) & =\left(1+\left[f^{\prime}(x)\right]^{2}\right)^{1 / 2} \frac{\partial G\left(\mathbf{r}, \mathbf{r}^{\prime}\right)}{\partial \mathbf{n}(\mathbf{r})}, \\
\psi(x) & =\left(1+\left[f^{\prime}(x)\right]^{2}\right)^{1 / 2} \frac{\partial u_{\mathrm{inc}}(\mathbf{r})}{\partial \mathbf{n}(\mathbf{r})},
\end{aligned}
$$

for $\mathbf{r}=\mathbf{s}(x)$ and $\mathbf{r}^{\prime}=\mathbf{s}\left(x^{\prime}\right)$, respectively. The limits of integration used in Eq. (10), which could otherwise be chosen arbitrarily as long as they delimit exactly a single period of $\partial \mathcal{S}$, are selected in our treatment in such a way that the singular term lies at the center of the domain of integration-thus avoiding the appearance of nearsingular integrands.

Once the unknown density $\phi$ in Eq. (10) has been determined, substitution of Eq. (9) and the spectral series, Eq. (8), into the scattered field expression, Eq. (5), gives rise to a plane wave expansion with direction vectors $\mathbf{K}_{n}$ $=\left(\alpha_{n}, \beta_{n}\right)$ for the field $u_{s}$ in the region $z>\max \{f(x)\}$,

$$
u_{s}^{+}(\mathbf{r})=\sum_{n=-\infty}^{\infty} b_{n} e^{i \mathbf{K}_{n} \cdot \mathbf{r}}
$$

with

$$
b_{n}=\frac{-i}{2 P \beta_{n}} \int_{0}^{P} \phi(x) e^{-i \mathbf{K}_{n} \cdot \mathbf{s}(x)} \mathrm{d} x .
$$

The quantity

$$
e_{n}=\frac{\operatorname{Re}\left(\beta_{n}\right)}{\beta}\left|b_{n}\right|^{2}
$$

which gives the fraction of the incident energy that is reflected in the $\mathbf{K}_{n}$ direction, is often referred to as the efficiency of the $n$th mode. The evanescent (nonpropagating) modes, for which $\operatorname{Re}\left(\beta_{n}\right)=0$, do not contribute to the far field. In particular, they carry no energy away from the surface, so that conservation of energy requires

$$
E=\sum_{\mathcal{U}} e_{n}=1
$$

where $\mathcal{U}$ is the (finite) set of indices corresponding to propagating modes:

$$
\mathcal{U}=\left\{n: \alpha_{n} \leqslant k\right\}
$$

The extent to which the energy balance condition (14) is satisfied by a numerical solution is often a good indicator of numerical accuracy. Although we include numerical energy-balance values $|1-E|$ among our numerical results, our ultimate accuracy checks result from evaluation, via convergence studies, of the maximum of the error in the physical quantities $e_{n}, n \in \mathcal{U}$.

\section{KERNEL DECOMPOSITION}

As mentioned in Section 1, our algorithm incorporates, as a key element, the decomposition, Eq. (2), of the kernel function $K$ in terms of smooth (analytic for analytic $f$ ) functions $K_{1}$ and $K_{2}$. A description of this important expression of the kernel is given in what follows.

The singularity of the kernel $K\left(x, x^{\prime}\right)$ is grasped easily by consideration of its series expansion Eq. (A1). In view of this formula and the identity ([10], Eq. 9.1.28) for the derivatives of the Hankel functions, we see that the kernel may be expressed in the form

$$
K\left(x, x^{\prime}\right)=-\frac{i k}{4} c\left(x, x^{\prime}\right) \frac{H_{1}^{(1)}\left[k r\left(x, x^{\prime}\right)\right]}{r\left(x, x^{\prime}\right)}+\mathcal{A}_{1}\left(x, x^{\prime}\right),
$$

with $c\left(x, x^{\prime}\right)=f(x)-f\left(x^{\prime}\right)-\left(x-x^{\prime}\right) f^{\prime}(x)$ and $r\left(x, x^{\prime}\right)=\left[\left(x-x^{\prime}\right)^{2}\right.$ $\left.+\left[f(x)-f\left(x^{\prime}\right)\right]^{2}\right]^{1 / 2}$. For $\left|x-x^{\prime}\right|<P$, all of the singular terms of the kernel are contained in the Hankel function of first order. In Eq. (15) $\mathcal{A}_{1}$ denotes the smooth (analytic) function

$$
\mathcal{A}_{1}\left(x, x^{\prime}\right)=\left(1+\left[f^{\prime}(x)\right]^{2}\right)^{1 / 2} \frac{\partial \mathcal{D}\left(\mathbf{r}, \mathbf{r}^{\prime}\right)}{\partial \mathbf{n}(\mathbf{r})},
$$

with

$$
\mathbf{r}=\mathbf{s}(x), \quad \mathbf{r}^{\prime}=\mathbf{s}\left(x^{\prime}\right)
$$

details concerning the function $\mathcal{D}$ and procedures for its numerical evaluation as well as that of its gradient are presented in Appendix A.

The Hankel function of unit order $H_{1}^{(1)}(z)=J_{1}(z)$ $+i Y_{1}(z)$ is singular for $z=0$. To proceed with the extraction of the singular logarithmic terms in the kernel, we consider the Neumann expansion for the $Y_{1}$ Bessel functions [10],

$$
\begin{aligned}
Y_{1}(z)= & -\frac{2}{z \pi} J_{0}(z)+\frac{2}{\pi}\left[\ln \left(\frac{z}{2}\right)+\gamma-1\right] J_{1}(z) \\
& -\frac{2}{\pi} \sum_{p=1}^{\infty} \frac{(-1)^{p}(2 p+1)}{p(p+1)} J_{2 p+1}(z),
\end{aligned}
$$

where $\gamma=0.57721566 \ldots$ is Euler's constant. In Eq. (17) we note the presence of a nonintegrable singularity at $z=0$, which is associated with the $J_{0}(z) / z$ term. It is well known (and easy to verify) that, while the gradient of the Green's function possesses such a singularity, the normal derivative is itself integrable. It is also easy to check that the only remaining singularity in $K$ is logarithmic, and that the kernel functions $K_{1}$ and $K_{2}$ appearing in Eq. (2) may be expressed in the form

$$
K_{1}\left(x, x^{\prime}\right)=\frac{k}{2 \pi} c\left(x, x^{\prime}\right) \frac{J_{1}\left(k r\left(x, x^{\prime}\right)\right)}{r\left(x, x^{\prime}\right)}
$$




$$
\begin{aligned}
K_{2}\left(x, x^{\prime}\right)= & \ln \left[\frac{k}{2} \sqrt{1+\left(\frac{f(x)-f\left(x^{\prime}\right)}{x-x^{\prime}}\right)^{2}}\right] K_{1}\left(x, x^{\prime}\right) \\
& +\mathcal{A}_{1}\left(x, x^{\prime}\right)-\frac{i k^{2}}{4} c\left(x, x^{\prime}\right) \\
& \times\left\{\mathcal{A}_{2}\left[k r\left(x, x^{\prime}\right)\right]-\frac{2 i}{\pi} \frac{J_{0}\left[k r\left(x, x^{\prime}\right)\right]}{\left[k r\left(x, x^{\prime}\right)\right]^{2}}\right\} .
\end{aligned}
$$

For convenience both in the notation and in the numerical evaluation of the function $K_{2}$, in Eq. (19) we have used the $\mathcal{A}_{2}$ function

$$
\mathcal{A}_{2}(z)=\frac{1}{z} H_{1}^{(1)}(z)+\frac{2 i}{z \pi}\left[\frac{1}{z} J_{0}(z)-\ln \left(\frac{z}{2}\right) J_{1}(z)\right]
$$

This definition is particularly useful when computing values of $K_{2}$ near $x=x^{\prime}$ : since $\mathcal{A}_{2}$ is analytic at $z=0$, we may evaluate this function accurately in a neighborhood of the origin by means of its Taylor series expansion-the leading order of which is $\mathcal{A}_{2}(0)=1 / 2+i(\gamma-1) / \pi$. Away from the origin, in turn, we compute $\mathcal{A}_{2}$ by explicit evaluation of all Hankel and Bessel functions in Eq. (20). As a simple exercise we note that the self-terms of the kernel functions are given by $K_{1}(x, x)=0$ and

$$
K_{2}(x, x)=\mathcal{A}_{1}(x, x)+\frac{1}{4 \pi} \frac{f^{\prime \prime}(x)}{1+\left[f^{\prime}(x)\right]^{2}} .
$$

It is important to note that, although the functions $K_{1}$ and $K_{2}$ are smooth for $\left|x-x^{\prime}\right|<P$-provided so is the surface $\partial \mathcal{S}$ - they are not periodic and, thus, a numerical procedure involving expansion of the functions $K_{1}$ and $K_{2}$ in Fourier series would give rise to limited accuracy. In what follows we use, instead, high-order-accurate expansions of these functions in Chebyshev series, and, after introducing our discretization of the unknown density $\phi$, we introduce an associated high-order integration algorithm for the integral in Eq. (10).

\section{NUMERICAL SOLUTION OF THE INTEGRAL EQUATIONS}

In this section we present a high-order solver for the second-kind integral equations (10). Our method incorporates (i) a high-order representation of the unknown integration density (Subsection 4.A) and (ii) high-order treatment of the resulting integrals (Subsection 4.B). The latter high-order treatment, in turn, is built upon (iii) integration weights that are computed accurately and efficiently by means of an asymptotic expansion as the number of integration points tends to infinity (Subsection 4.C).

\section{A. Floquet Expansions and Associated Discretizations} It is well-known that the surface density $\phi$ may be represented as a Floquet series

$$
\phi(x)=\sum_{n=-\infty}^{\infty} \hat{\phi}_{n} e^{i \alpha_{n} x}
$$

Denoting by $\widetilde{\phi}_{N}(x)=\Sigma_{n=-N}^{N} \hat{\phi}_{n} e^{i \alpha_{n} x}$ a truncated Floquet series, for $\phi \in \mathcal{C}^{m+1}$, the truncation error is $\left|\phi(x)-\widetilde{\phi}_{N}(x)\right|$ $=\mathcal{O}\left(N^{-m}\right)$ uniformly in the interval $[-P / 2, P / 2]$ : for $\phi$ $\in \mathcal{C}^{\infty}$, the Floquet series converges faster than $\mathcal{O}\left(N^{-m}\right)$ for any positive integer $m$-i.e., it achieves superalgebraic convergence. Our algorithm uses a truncated Floquet expansion together with superalgebraically convergent quadratures, and it can thus achieve itself superalgebraically high accuracy.

Using a finite Floquet expansion,

$$
\phi_{N}(x)=\sum_{n=-N}^{N} a_{n} e^{i \alpha_{n} x}
$$

to approximate the unknown $\phi$, the integral equation (10) is approximated by the equation

$$
\psi(x)=\sum_{n=-N}^{N} a_{n}\left(\frac{1}{2} e^{i \alpha_{n} x} \pm W_{n}(x)\right),
$$

for the coefficients $a_{n}$, where

$$
W_{n}(x)=\int_{x-P / 2}^{x+P / 2}\left\{K_{1}\left(x, x^{\prime}\right) \ln \left|x-x^{\prime}\right|+K_{2}\left(x, x^{\prime}\right)\right\} e^{i \alpha_{n} x^{\prime}} \mathrm{d} x^{\prime} .
$$

Point matching Eq. (23) at $2 N+1$ equispaced values of $x$ $\in[-P / 2, P / 2]$ yields a well-posed "spatial collocation" system of equations for the Floquet amplitudes $a_{n}$. An alternative linear equation for the coefficients $a_{n}$ is obtained by considering the Floquet series coefficients $d_{n}$ of $\psi$; in this case, the finite dimensional linear system for the coefficients $a_{n}$ is given by

$$
d_{m}=\frac{1}{2} a_{m} \pm \sum_{n=-N}^{N} a_{n} B_{m, n}
$$

where

$$
B_{m, n}=\frac{1}{P} \int_{0}^{P} W_{n}(x) e^{-i \alpha_{m} x} \mathrm{~d} x .
$$

It is readily seen that the second formulation above arises from the first one via a $(2 N+1)$ point discrete Fourier transform. In Appendix B we briefly compare the performance of algorithms arising from these two linear systems; in short, our experiments indicate that the "spectral testing" approach embodied in Eq. (25) can give rise to somewhat more accurate solutions-by up to one or two digits in some cases - than the method obtained through spatial collocation of Eq. (23). With the exception of the column labeled spatial collocation in Table 9, all of the numerical results presented in this paper were obtained by means of the spectral testing approach.

Once the approximate solution $\phi_{N}$ has been found, approximate values of the amplitudes $b_{n}$ [and thus, via Eq. (13), of the efficiencies $e_{n}$ ] result from Eq. (12): substitution of the series expansion Eq. (22) into Eq. (12) yields 


$$
b_{n}=-\frac{i}{2} \sum_{m=-N}^{N} c_{m, n} a_{m}
$$

where the coefficients $c_{m, n}$, which are given by

$$
c_{m, n}=\frac{1}{P} \int_{0}^{P} e^{2 m \pi i x / P}\left\{\frac{e^{-2 n \pi i x / P-i \beta_{n} f(x)}}{\beta_{n}}\right\} \mathrm{d} x,
$$

can be obtained accurately and efficiently by means of the fast Fourier transform (FFT) of the functions in braces; in our implementation, these coefficients are obtained from a $2 N+1$ point FFT for each index $n \in \mathcal{U}$. We now turn to the needed efficient evaluation of the integrals (24).

\section{B. Efficient Evaluation of the Functions $W_{n}$}

Using the coordinate transformation $y=2\left(x^{\prime}-x\right) / P$ we may write

$$
W_{n}(x)=\frac{P}{2} e^{i \alpha_{n} x} \int_{-1}^{1}\left\{\widetilde{K}_{1}(x, y) \ln |y|+\widetilde{K}_{2}(x, y)\right\} e^{i n \pi y} \mathrm{~d} y,
$$

where

$$
\begin{aligned}
\widetilde{K}_{1}(x, y)= & K_{1}\left(\begin{array}{c}
P \\
x, \frac{1}{2} y+x
\end{array}\right) e^{i \alpha P y / 2}, \\
\widetilde{K}_{2}(x, y)= & \ln \left(\frac{P}{2}\right) \widetilde{K}_{1}(x, y) \\
& +K_{2}\left(x, \frac{P}{2} y+x\right) e^{i \alpha P y / 2} .
\end{aligned}
$$

To evaluate $W_{n}$ we expand the functions, Eqs. (29) and (30), in truncated Chebyshev series,

$$
\widetilde{K}_{j}(x, y) \approx \frac{1}{2} A_{0}^{(j)}(x)+\sum_{m=1}^{M} A_{m}^{(j)}(x) T_{m}(y), \quad j=1,2 .
$$

Such expansions converge rapidly: for smooth $f$ the functions $\widetilde{K}_{j}$ are themselves smooth, and the approximation error in Eq. (31) tends to zero faster than $\mathcal{O}\left(M^{-m}\right)$ for all positive integers $m$ [11].

For each given value of $x$, the series coefficients are produced by the formula $[11,12]$

$$
A_{m}^{(j)}(x)=\frac{2}{\pi} \int_{-1}^{1} \frac{\widetilde{K}_{j}(x, y) T_{m}(y)}{\sqrt{1-y^{2}}} \mathrm{~d} y, \quad m=0,1,2, \ldots
$$

Equations (31) and (32) constitute the Chebyshev transform pair of the function $\widetilde{K}_{j}$. As is well known, with the change of variables $y=\cos \theta$ we have $T_{m}(\cos \theta)=\cos m \theta$, and the Chebyshev coefficients in Eq. (32) are given by the cosine transform of $\widetilde{K}_{j}(x, \cos \theta)$ in $\theta$ space. It follows that, for each $x$, the Chebyshev coefficients can be produced in $\mathcal{O}(M \log M)$ operations using a fast cosine transform.

The functions $W_{n}$ are thus approximated by

$$
W_{n}(x) \approx \frac{P}{2} e^{i \alpha_{n} x} \sum_{j=1}^{2}\left\{\frac{1}{2} A_{0}^{(j)}(x) E_{0, n}^{(j)}+\sum_{m=1}^{M} A_{m}^{(j)}(x) E_{m, n}^{(j)}\right\}
$$

where the "integration weights" $E_{m, n}^{(j)}, j=1,2$, are given by

$$
E_{m, n}^{(1)}=\int_{-1}^{1} \ln |x| T_{m}(x) e^{i n \pi x} \mathrm{~d} x, \quad E_{m, n}^{(2)}=\int_{-1}^{1} T_{m}(x) e^{i n \pi x} \mathrm{~d} x .
$$

An accurate and efficient method for evaluation of the weights, Eq. (34), is presented in Subsection 4.C.

\section{Efficient Evaluation of Integration Weights $\boldsymbol{E}_{m, n}^{(j)}$}

\section{Basic Strategy}

To evaluate the weights, Eq. (34), we use the change of variables $x=\cos \theta$ together with the sum of the first $Q$ terms of the exponentially convergent expansion ([10], Eqs. 9.1.44 and 9.1.45)

$$
e^{i z \cos \theta}=J_{0}(z)+2 \sum_{k=1}^{\infty} i^{k} J_{k}(z) T_{k}(\cos \theta),
$$

and the relations [12] $T_{k}(\cos \theta)=\cos k \theta$ and $T_{m}(x) T_{k}(x)$ $=\left[T_{m+k}(x)+T_{|m-k|}(x)\right] / 2$ ( $m$ and $k$ nonnegative integers); interchanging the order of summation and integration in Eq. (34) we thus obtain

$$
\begin{aligned}
E_{2 m, n}^{(j)}= & J_{0}(n \pi) I_{2 m}^{(j)}+\sum_{k=1}^{[Q / 2]}(-1)^{k} J_{2 k}(n \pi)\left\{I_{2(m+k)}^{(j)}+I_{2|m-k|}^{(j)}\right\}, \\
E_{2 m+1, n}^{(j)}= & i \sum_{k=0}^{[Q / 2]}(-1)^{k} J_{2 k+1}(n \pi)\left\{I_{2(m+k+1)}^{(j)}+I_{2 \mid m-k}^{(j)}\right\}, \quad j=1,2,
\end{aligned}
$$

where we have set

$$
I_{2 n}^{(1)}=\int_{-1}^{1} \ln |y| T_{2 n}(y) \mathrm{d} y, \quad I_{2 n}^{(2)}=\frac{2}{1-4 n^{2}} .
$$

The series truncation parameter $Q$ that, to maintain accuracy, is chosen as a function of the argument of the Bessel functions, is determined by means of a scheme that we outline in Subsection 4.C.3. From the second equation of Eq. (38) it is clear that $\left|I_{2 n}^{(2)}\right|<1$; it is also possible to show that $\left|I_{2 n}^{(1)}\right|<1$; we thus expect the series, Eqs. (36) and (37), to converge at least as quickly as the corresponding cylindrical wave expansions and not affect our choice of truncation parameter $Q$.

2. Evaluation of the Coefficients $I_{2 n}^{(1)}$ in Eq. (38)

It is easy to check that, letting

$$
L_{2 n+1}=(-1)^{n} \int_{0}^{\pi / 2} \ln (\sin \theta) \cos (2 n+1) \theta \mathrm{d} \theta,
$$

we have $I_{2 n}^{(1)}=L_{2 n+1}-L_{2 n-1}$ for $n \geqslant 0$. Replacing $\cos (2 n$ +1) $\theta$ by its Chebyshev series and using the identity ([13], Eq. 4.387), we obtain 


$$
\begin{aligned}
L_{2 n+1}= & -\frac{\pi}{8}(2 n+1) \sum_{m=0}^{n} \frac{(-1)^{m}(n+m) !}{(n-m) ![\Gamma(m+3 / 2)]^{2}} \\
& \times[\Psi(m+3 / 2)-\Psi(1 / 2)], \quad n \geqslant 0,
\end{aligned}
$$

where $\Psi$ is the digamma function [10]. We have found that the use of Eq. (40) leads to large subtractive cancellations for moderate to large values of $n$. We therefore restrict the use of Eq. (40) to small values of $n$, as detailed in Subsection 4.C.3, and for larger values of $n$ we produce $L_{2 n+1}$ by means of an asymptotic series, which we derive in what follows.

To produce the desired asymptotic expansion for $L_{2 n+1}$ we multiply and divide by $\theta$ the argument of the logarithm in Eq. (39), and thus express $L_{2 n+1}$ in the form

$$
\begin{aligned}
L_{2 n+1}= & \frac{1}{2 n+1} \ln \left(\frac{\pi}{2}\right)+\frac{(-1)^{n+1}}{2 n+1} \operatorname{Si}\left[\frac{\pi(2 n+1)}{2}\right] \\
& +(-1)^{n} \int_{0}^{\pi / 2} \ln \left(\frac{\sin \theta}{\theta}\right) \cos (2 n+1) \theta \mathrm{d} \theta
\end{aligned}
$$

where $\operatorname{Si}(z)=\int_{0}^{z} \sin (t) / t \mathrm{~d} t$ is the sine integral ([10], Eq. 5.2.1).

An asymptotic expansion is readily available for the sine integral: from the equations ([10], Eqs. 5.2.8 and 5.2.35) we easily obtain

$$
\begin{aligned}
\operatorname{Si}\left[\frac{\pi(2 n+1)}{2}\right]= & \frac{\pi}{2}+\sum_{m=1}^{M} \frac{(-1)^{n+m}(2 m-1) !}{(2 n+1)^{2 m}}\left(\frac{2}{\pi}\right)^{2 m} \\
& +\mathcal{O}\left(\frac{1}{(2 n+1)^{2 M+3}}\right) .
\end{aligned}
$$

To derive an asymptotic expansion for the integral in Eq. (41), in turn, we resort to integration by parts. The derivatives of the function $\ln [\sin (\theta) / \theta]$ at $\theta=0$ and $\theta=\pi / 2$ may be obtained easily from equations ([10], Eqs. 4.3.71 and 4.3.72). Thus, integrating by parts the second term in Eq. (41) we obtain

$$
\begin{aligned}
(-1)^{n} \int_{0}^{\pi / 2} \ln \left(\frac{\sin \theta}{\theta}\right) \cos (2 n+1) \theta \mathrm{d} \theta \\
=-\frac{1}{2 n+1} \ln \left(\frac{\pi}{2}\right)+\sum_{m=1}^{M} \frac{(-1)^{m}(2 m-1) !}{(2 n+1)^{2 m+1}}\left(\frac{2}{\pi}\right)^{2 m} \\
\quad+\sum_{m=1}^{M} \frac{2^{2 m-1}\left(2^{2 m}-1\right) B_{2 m}}{m(2 n+1)^{2 m+1}}+\mathcal{O}\left(\frac{1}{(2 n+1)^{2 M+3}}\right),
\end{aligned}
$$

where $B_{m}$ are the Bernoulli numbers ([10], Eq. 23.1.2). The Bernoulli numbers can be evaluated in a number of ways; the small number of such quantities we need can be obtained directly from [10], Table 23.2, or, even more simply, from the Maple implementation of the Bernoulli numbers. We thus obtain

$$
\begin{aligned}
L_{2 n+1}= & \frac{(-1)^{n+1} \pi}{2(2 n+1)}+\sum_{m=1}^{M} \frac{2^{2 m-1}\left(2^{2 m}-1\right) B_{2 m}}{m(2 n+1)^{2 m+1}} \\
& +\mathcal{O}\left(\frac{1}{(2 n+1)^{2 M+3}}\right),
\end{aligned}
$$

Forming $I_{2 n}^{(1)}=L_{2 n+1}-L_{2 n-1}$ from Eq. (44) and expanding $(2 n+1)^{-2 m-1}-(2 n-1)^{-2 m+1}$ in powers of $(2 n)^{-2 l}$ for each $m \geqslant 1$ leads to

$$
I_{2 n}^{(1)}=\frac{2 n \pi(-1)^{n+1}}{4 n^{2}-1}-2 \sum_{l=1}^{l_{\max }} \frac{(-1)^{l} \alpha_{2 l}}{(2 n)^{2 l}}+\mathcal{O}\left(\frac{1}{(2 n)^{2 l_{\text {max }}+2}}\right),
$$

(an expression which in practice we found to be slightly more accurate than the direct subtraction $L_{2 n+1}-L_{2 n-1}$ ), where the integer coefficients $\alpha_{2 l}$ for $l=0, \ldots, 12$ are given in Table 1 . With $l_{\max }=12$ this expansion correctly produces $I_{2 n}^{(1)}$ for $n \geqslant 12$ to full double-precision accuracy. [Indeed the simple expansion

$$
I_{2 n}^{(1)}=-\frac{2 n \pi(-1)^{n}}{4 n^{2}-1}-\frac{6}{(2 n)^{4}}+\mathcal{O}\left(\frac{1}{(2 n)^{8}}\right)
$$

is sufficient to produce full double-precision results for all $n \geqslant 100$.] For $n \leqslant 11$, finally, we obtain the $I_{2 n}=L_{2 n+1}$ $-L_{2 n-1}$ values by means of the expression Eq. (40), which yields accurate results for small values of $n$; these few values were obtained once and hard coded as part of our implementation.

\section{Parameter Selection and Other Practical} Considerations

Accurate values of the weights, Eq. (34), can be obtained efficiently by means of the procedures described in Subsections 4.C.1 and 4.C.2, together with a few additional details that we present in what follows. We first point out that, since $T_{m}(-x)=(-1)^{m} T_{m}(x)$, it follows from Eq. (34) that $E_{m,-n}^{(j)}=(-1)^{m} E_{m, n}^{(j)}, j=1,2$; it thus suffices to evaluate and store $E_{m, n}^{(j)}$ for nonnegative values of $n$.

The sequence of Bessel functions values $J_{k}(n \pi), k$ $=0,1, \ldots, Q+1$ that are required to evaluate the sums Eq. (36) and (37), in turn can be efficiently computed, for each

Table 1. Coefficients $\alpha_{2 \ell}, \ell=0, \ldots, 12$, Used in Expansion (45)

\begin{tabular}{cr}
\hline$\ell$ & \multicolumn{1}{c}{$\alpha_{2 l}$} \\
\hline 1 & 0 \\
2 & 3 \\
3 & 0 \\
4 & 63 \\
5 & 1320 \\
6 & 49,203 \\
7 & $2,653,560$ \\
8 & $196,707,423$ \\
9 & $19,194,804,720$ \\
10 & $2,385,684,870,723$ \\
11 & $367,985,503,366,800$ \\
12 & $68,980,888,889,771,103$ \\
\hline
\end{tabular}




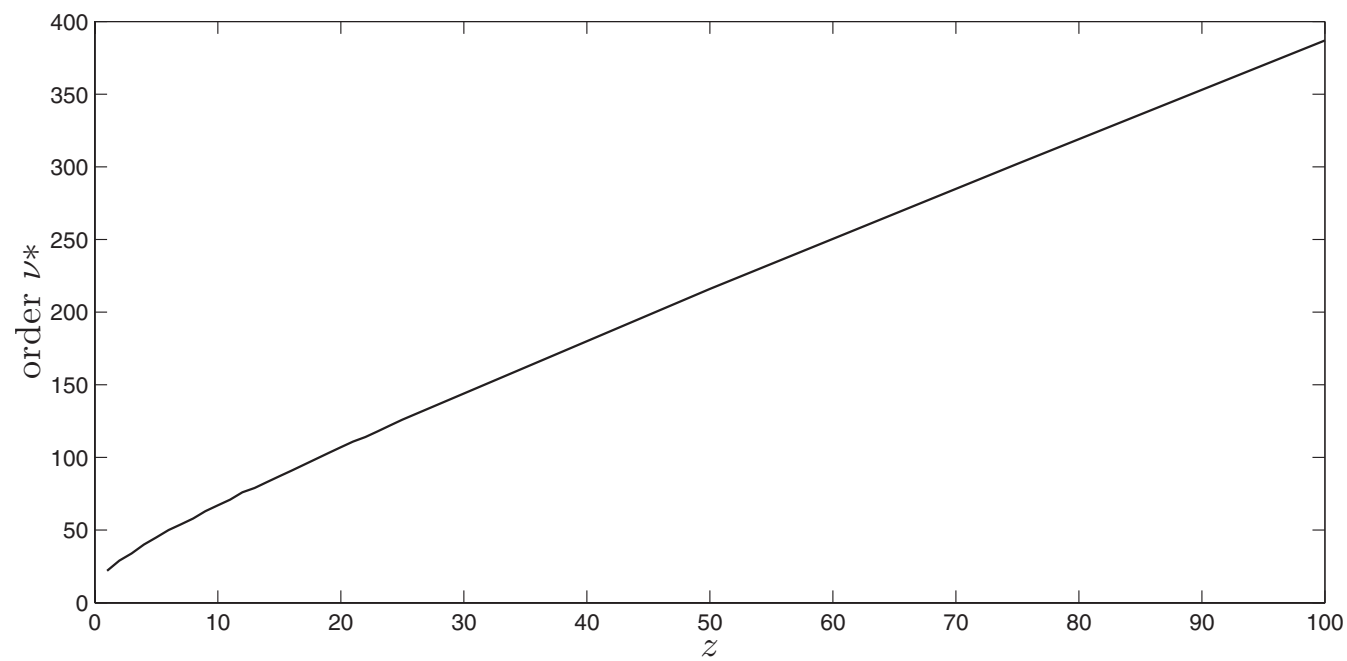

Fig. 1. Order of Bessel function such that $J_{\nu}(\pi z)=\mathcal{O}\left(10^{-16}\right)$ for all $\nu \geqslant \nu^{*}$. The curve defining $\nu^{*}$ is well-approximated using low-order polynomials.

fixed value of $n$, through an application of the DBESJ routine in the Slatec library [14]. The DBESJ routine computes an accurate estimate for $J_{Q+1}(n \pi)$ and then produces the remaining members of the sequence by means of a stable backwards recurrence.

Our final point in this section concerns the selection of adequate values of the truncation parameter $Q$ introduced in Subsection 4.C.1. To select this parameter it is sufficient to evaluate, as a function of $z$, the Bessel function order $\nu^{*}$ such that for all $\nu>\nu^{*}$ we have $J_{\nu}(\pi z)=\mathcal{O}(\epsilon)$; in this work we have used the value $\epsilon=10^{-16}$, which is suitable for full double-precision calculations. We found it expedient to obtain numerically the curve $\nu^{*}(z)$ (Fig. 1) by direct evaluation of Bessel functions $J_{\nu}(\pi z)$ for sequences of values of $\nu$ and for a sufficiently fine $z$ mesh, followed by interpolation of the $\nu^{*}(z)$ by a set of low degree polynomials,

$$
\nu^{*}=a_{0}^{(i)}+a_{1}^{(i)} z+a_{2}^{(i)} z^{2}
$$

each one valid in a subdomain $z \in\left(z_{i}, z_{i+1}\right)$. Once a suitable approximation of the function $\nu^{*}(z)$ has been obtained, we set $Q=\left[\nu^{*}\right]+1$, where $[x]$ is the greatest integer not exceeding $x$. Values of the coefficients $a_{0}^{(i)}, a_{1}^{(i)}$, and $a_{2}^{(i)}$ are given in Table 2.

Table 2. Parameters Defining Interpolants of the Function $\nu^{*}(z)^{a}$

\begin{tabular}{ccccc}
\hline$z_{i}$ & $z_{i+1}$ & $a_{0}^{(i)}$ & $a_{1}^{(i)}$ & $a_{2}^{(i)}$ \\
\hline 1 & 25 & $1.9349 \times 10^{1}$ & 5.0731 & $-3.4114 \times 10^{-2}$ \\
25 & 200 & $3.8393 \times 10^{1}$ & 3.5681 & $-8.1905 \times 10^{-4}$ \\
200 & 1000 & $6.8173 \times 10^{1}$ & 3.2753 & $-4.8158 \times 10^{-5}$ \\
1000 & 2000 & $9.4061 \times 10^{1}$ & 3.2126 & $-1.0606 \times 10^{-5}$ \\
2000 & 4000 & $1.2388 \times 10^{2}$ & 3.1820 & $-2.6515 \times 10^{-6}$ \\
4000 & 6000 & $1.5245 \times 10^{2}$ & 3.1684 & $-9.9068 \times 10^{-7}$ \\
6000 & 8000 & $1.8265 \times 10^{2}$ & 3.1591 & $-2.7473 \times 10^{-7}$ \\
\hline
\end{tabular}

${ }^{a}$ For $z>8000$ we use the linear relationship $\nu^{*}=210+3.1535 z$, which leads to slight overestimates of the truncation index $Q=\left[\nu^{*}(z)\right]+1$.

\section{RESULTS AND DISCUSSION}

In what follows we present a variety of numerical results produced by the algorithms described in the previous sections. In particular, we present results for gratingdiffraction problems in the resonance regime (heights and periods up to a few wavelengths) as well as very challenging grating scattering problems, featuring very deep gratings, very high frequencies, and nearly glacing incidences: some of these problems are significantly more demanding than some of the most complex grating-diffraction problems that have ever been considered in the literature. In all cases high accuracies can be produced from the algorithms introduced in this text; in each example the error is estimated by means of the expression

$$
\text { error }=\max _{n}\left|e_{n}-e_{n}^{*}\right|
$$

where $e_{n}^{*}$ is the $n$-order efficiency resulting from use of a large number $N=N^{*}$ of discretization points; the values of $N^{*}$ used in each case are listed together with the corresponding numerical results. Note that this error quantifier does not equal the "energy balance" error estimator, given by $|1-E|=\left|1-\Sigma_{n} e_{n}\right|$, that is used frequently in the literature (e.g. [3]). For the sake of completeness, our tables of numerical results include the energy balance error estimator as well. All computations presented in this section were performed on an Intel Xeon $3 \mathrm{GHz}$ processor. Solutions of all linear systems were obtained by means of the LU-based direct solvers provided in the LAPACK linear algebra package [15].

To demonstrate the rapid high-order convergence provided by our algorithm, in Table 3 we compare the results of our method to those provided by the highly accurate high-order algorithm introduced in [16] for the profile used in that contribution: $f(x)=d+a \sin x$. With reference to Table 3 in [16], we note that the algorithm [16] requires $N=128$ to achieve the same accuracy as the present method does with $N=10$ : clearly, the combination of Floquet expansions and spectral weight-based integration provides high-order accuracy in a very effective manner. 
Table 3. Results Provided by Our Method for the TE Test Case Considered in Table 3 of $[16]^{a}$

\begin{tabular}{cccccc}
\hline & \multicolumn{2}{c}{$\theta=\pi / 2$} & \multicolumn{2}{c}{$\theta=\pi / 6$} \\
\cline { 2 - 5 }$N$ & $M$ & $|1-E|$ & Error & $|1-E|$ & Error \\
\hline 4 & $2^{6}$ & $1.26 \times 10^{-4}$ & $9.56 \times 10^{-4}$ & $1.67 \times 10^{-4}$ & $6.66 \times 10^{-4}$ \\
6 & $2^{6}$ & $1.26 \times 10^{-7}$ & $3.14 \times 10^{-7}$ & $1.39 \times 10^{-7}$ & $1.03 \times 10^{-7}$ \\
8 & $2^{6}$ & $8.59 \times 10^{-10}$ & $1.45 \times 10^{-10}$ & $1.43 \times 10^{-10}$ & $1.78 \times 10^{-10}$ \\
10 & $2^{6}$ & $1.32 \times 10^{-13}$ & $1.28 \times 10^{-13}$ & $2.11 \times 10^{-13}$ & $1.36 \times 10^{-13}$ \\
\hline
\end{tabular}

${ }^{a}$ Our results for the TM case exhibit nearly identical behavior. Our algorithm's parameters are $k=1.25 / 0.546, d=3, a=0.7, M_{v}=129$ for all the cases considered in this table. The reference solution was computed with the same parameters listed in each row of the table and $N^{*}=30$. The execution times required by our code for these tests are very small; for the case $N=10$, for example, the execution time was $t_{\text {exe }}$ $=0.43 \mathrm{~s}$.

In what follows we present data sets corresponding to the standard rough-surface sinusoidal test surface

$$
f(x)=a \cos \left(\frac{2 \pi x}{P}\right) .
$$

Code parameters, computation times, and resulting accuracies for various problems in the classical resonance regime are presented in Table 4 . We only present results corresponding to the TE problem; results corresponding to the TM case are nearly identical. In all cases considered, near machine precision accuracies are obtained with a small sampling of the integral operator and very small computational times.

To demonstrate the robustness of our solver, further, we consider several cases that, to the best of our knowledge, could not previously be successfully tackled on desktop personal computers. First, in Table 5 we consider problems with incidence angle fixed at a moderate value and with a moderate value of the frequency (supporting 40 propagating modes); the amplitude of the profile, in turn, is allowed to vary between 4 and 20 (i.e., the peak-totrough height varies between 8 and 40 times the period). In each case near machine precision accuracies are produced. The case $a / P=4$ was previously considered in [3]; the most accurate method presented in that reference requires times of the order of 662 min on a Sparc 20 workstation to achieve four-digit accuracies for this problem. Our method, in contrast, achieves nearly double precision accuracies in computing times of the order of $10 \mathrm{~min}$ in this case. Errors of the order of $10^{-12}$ to $10^{-10}$ in Table 5 result from roundoff accumulation in the evaluation of integration weights.

In Table 6 we demonstrate the stability of our solver for near-grazing configurations, even with a rather small wavelength. In each case shown, the algorithm resolves 200 propagating modes, and it achieves full doubleprecision results up to $\theta=0.01^{\circ}$. Note that the case $\theta$ $=0.01^{\circ}$ required $M_{v}=4097$; further refinement of the Green's function is required when computing the Veysoglu integrals for very shallow incidence (see Appendix A). For these cases, some deterioration in the accuracy of the solution occurs due to a subtractive cancellation in evaluating terms of the form $k(1-\cos \theta)$. This difficulty is easily bypassed by setting $1-\cos \theta=2 \sin ^{2}(\theta / 2)$ in the Veysoglu integrals and $\beta_{0}=k \sin \theta$ in Eqs. (8) and (12). In Table 7, in turn, we demonstrate the stability of our solver for highfrequency problems. In each case, near machine precision accuracies are obtained for both TE and TM problems.

Table 4. Code Parameters, Computation Times, and Resulting Accuracies for Various Problems in the Classical Resonance Regime, TE Problem (TM Results Are Nearly Identical) ${ }^{a}$

\begin{tabular}{ccccccccccccc}
\hline & \multicolumn{9}{c}{$P / \lambda=1.0$} & \multicolumn{3}{c}{$P / \lambda=2.0$} \\
\cline { 2 - 11 } & $N$ & $M$ & Error & $t_{\text {exe }}$ & $N$ & $M$ & Error & $t_{\text {exe }}$ & $N$ & $M$ & Error & $t_{\text {exe }}$ \\
\hline 0.5 & 15 & $2^{7}$ & $1.3 \times 10^{-14}$ & 0.67 & 20 & $2^{7}$ & $1.0 \times 10^{-14}$ & 0.74 & 30 & $2^{7}$ & $1.0 \times 10^{-14}$ & 1.02 \\
1.0 & 25 & $2^{7}$ & $3.9 \times 10^{-12}$ & 0.78 & 25 & $2^{8}$ & $2.2 \times 10^{-13}$ & 1.32 & 45 & $2^{8}$ & $1.8 \times 10^{-14}$ & 2.30 \\
1.5 & 35 & $2^{8}$ & $4.4 \times 10^{-14}$ & 1.82 & 35 & $2^{8}$ & $3.0 \times 10^{-14}$ & 1.64 & 55 & $2^{8}$ & $2.1 \times 10^{-12}$ & 2.45 \\
2.0 & 65 & $2^{8}$ & $1.0 \times 10^{-14}$ & 3.21 & 65 & $2^{8}$ & $1.0 \times 10^{-14}$ & 2.84 & 70 & $2^{9}$ & $5.2 \times 10^{-13}$ & 5.96 \\
\hline
\end{tabular}

${ }^{a}$ The code execution time $t_{\text {exe }}$ is measured in seconds. The reference solution was computed with the same parameters listed in each row of the table and $N^{*}=80$. In all examples presented here, the value $M_{v}=129$ was used for the evaluation of the Green's function; see Appendix A.

Table 5. Code Performance for Various Surface Heights $a$ With Parameters and Resulting Accuracy and Energy Balance for Both Cases of TE and TM Scattering from Surface Profile $f(x)=a \cos (2 \pi x / P)$ with $\lambda=0.05 P$ and $\theta=7 \pi / 18^{a}$

\begin{tabular}{ccccccccc}
\hline$a / P$ & $N$ & $M$ & $N^{*}$ & Error (TE) & $|1-E|(\mathrm{TE})$ & Error (TM) & $|1-E|(\mathrm{TM})$ & $t_{\text {exe }}$ \\
\hline 4 & 550 & $2^{11}$ & 700 & $7.35 \times 10^{-13}$ & $5.73 \times 10^{-12}$ & $7.58 \times 10^{-13}$ & $1.09 \times 10^{-12}$ & 10 \\
8 & 1050 & $2^{12}$ & 1200 & $6.14 \times 10^{-12}$ & $1.48 \times 10^{-11}$ & $7.52 \times 10^{-11}$ & $5.19 \times 10^{-11}$ & 64 \\
12 & 1600 & $2^{13}$ & 1700 & $1.49 \times 10^{-11}$ & $4.00 \times 10^{-12}$ & $3.75 \times 10^{-11}$ & $8.28 \times 10^{-11}$ & 236 \\
16 & 2100 & $2^{13}$ & 2200 & $3.59 \times 10^{-11}$ & $8.72 \times 10^{-11}$ & $5.74 \times 10^{-11}$ & $2.83 \times 10^{-11}$ & 392 \\
20 & 2600 & $2^{13}$ & 2700 & $9.80 \times 10^{-11}$ & $3.51 \times 10^{-11}$ & $2.05 \times 10^{-10}$ & $1.55 \times 10^{-10}$ & 570 \\
\hline
\end{tabular}

${ }^{a}$ In each case there are 40 propagating modes. The code execution time $t_{\text {exe }}$ is measured in minutes. In all examples presented here we use $M_{v}=1025$ to compute the Green's function; see Appendix A. 
Table 6. Code Performance for Grazing Incidence Problem: Incidence Angles $\theta$ (Measured in Degrees from Horizontal) with Parameters and Resulting Accuracies for Both Cases of TE and TM Scattering from Surface Profile $f(x)=a \cos (2 \pi x / P)$ with $a=P$ and $\lambda=0.01 P^{a}$

\begin{tabular}{ccccccccc}
\hline $\begin{array}{c}\theta \\
(\mathrm{deg})\end{array}$ & $N$ & $M$ & $M_{v}$ & Error (TE) & $|1-E|(\mathrm{TE})$ & Error (TM) & $|1-E|(\mathrm{TM})$ & $t_{\text {exe }}$ \\
\hline 1.00 & 800 & $2^{12}$ & $2^{10}+1$ & $3.00 \times 10^{-14}$ & $2.84 \times 10^{-14}$ & $2.61 \times 10^{-14}$ & $2.38 \times 10^{-14}$ & 50 \\
0.10 & 800 & $2^{12}$ & $2^{10}+1$ & $3.09 \times 10^{-15}$ & $2.32 \times 10^{-13}$ & $4.80 \times 10^{-14}$ & $2.35 \times 10^{-13}$ & 50 \\
0.01 & 800 & $2^{12}$ & $2^{12}+1$ & $2.47 \times 10^{-13}$ & $7.58 \times 10^{-14}$ & $1.21 \times 10^{-12}$ & $1.45 \times 10^{-12}$ & 72 \\
\hline
\end{tabular}

${ }^{a}$ In each case there are 200 propagating modes. The code execution time $t_{\text {exe }}$ is measured in minutes. The reference solution was computed with the same parameters listed in each row of the table and $N^{*}=900$. For cases $\theta=1.00^{\circ}$ and $0.10^{\circ}$ we use $M_{v}=1025$, while for $\theta=0.01^{\circ}$ we use $M_{v}=4097$; see Appendix A.

Table 7. Code Performance for Various Incidence Wavelengths $\lambda$ with Parameters and Resulting Accuracies for Both Cases of TE and TM Scattering from Surface Profile $f(x)=a \cos (2 \pi x / P)$ with $a=P$ and $\theta=\pi / 4^{a}$

\begin{tabular}{ccccccccc}
\hline$\lambda / P$ & $N$ & $M$ & $N^{*}$ & Error (TE) & $|1-E|(\mathrm{TE})$ & Error $(\mathrm{TM})$ & $|1-E|(\mathrm{TM})$ & $t_{\mathrm{exe}}$ \\
\hline 0.100 & 100 & $2^{9}$ & 200 & $1.40 \times 10^{-14}$ & $2.22 \times 10^{-16}$ & $1.30 \times 10^{-14}$ & $1.77 \times 10^{-14}$ & 0.25 \\
0.050 & 200 & $2^{10}$ & 300 & $2.40 \times 10^{-14}$ & $1.95 \times 10^{-14}$ & $2.32 \times 10^{-14}$ & $5.22 \times 10^{-14}$ & 1.3 \\
0.010 & 750 & $2^{12}$ & 900 & $2.90 \times 10^{-14}$ & $7.69 \times 10^{-14}$ & $4.85 \times 10^{-14}$ & $1.60 \times 10^{-13}$ & 45 \\
0.005 & 1500 & $2^{13}$ & 1600 & $6.99 \times 10^{-14}$ & $1.43 \times 10^{-13}$ & $7.59 \times 10^{-14}$ & $5.27 \times 10^{-13}$ & 221 \\
\hline
\end{tabular}

${ }^{a}$ The number of propagating modes in the above examples are 20, 40, 200, and 400. This is a challenging problem in the resonance (and approaching high frequency) regime. The code execution time $t_{\mathrm{exe}}$ is measured in minutes. In all examples presented here we use $M_{v}=1025$ to compute the Green's function; see Appendix A.

Table 8. Convergence Results for a Problem of Scattering by the Composite Surface Depicted in Fig. $2^{a}$

\begin{tabular}{cllllll}
\hline$N$ & $M$ & Error $(\mathrm{TE})$ & $|1-E|(\mathrm{TE})$ & Error $(\mathrm{TM})$ & $|1-E|(\mathrm{TM})$ & $t_{\text {exe }}$ \\
\hline 150 & $2^{10}$ & $2.14 \times 10^{-4}$ & $1.03 \times 10^{-3}$ & $1.11 \times 10^{-1}$ & $8.67 \times 10^{-1}$ \\
175 & $2^{10}$ & $3.13 \times 10^{-5}$ & $4.94 \times 10^{-5}$ & $1.90 \times 10^{-4}$ & $2.80 \times 10^{-5}$ \\
200 & $2^{10}$ & $2.67 \times 10^{-9}$ & $3.35 \times 10^{-9}$ & $7.13 \times 10^{-9}$ & $1.66 \times 10^{-8}$ \\
225 & $2^{10}$ & $1.30 \times 10^{-14}$ & $2.89 \times 10^{-14}$ & $2.06 \times 10^{-14}$ & $1.42 \times 10^{-14}$ \\
\hline
\end{tabular}

${ }^{a}$ Incidence data $\lambda=0.05 P, \theta=\pi / 4 ; 40$ propagating modes. The code execution time $t_{\text {exe }}$ is measured in seconds. The reference solution was computed using $N^{*}=400$. In all examples presented in this table the value $M_{v}=257$ was used for the evaluation of the periodic Green's function; see Appendix A.

Finally we present data sets corresponding to the composite surface

$$
f(x)=-2\left[\sin (x)+\frac{1}{2} \sin (2 x)+\frac{1}{3} \sin (3 x)+\frac{1}{4} \sin (4 x)\right] .
$$

Accuracies and code execution times are listed in Table 8. Clearly, the present algorithm is applicable to general periodic smooth surfaces.
In summary, we have introduced a rough-surface scattering solver that gives rise to superalgebraic convergence-faster than $\mathcal{O}\left(N^{-m}\right)$ and $\mathcal{O}\left(M^{-m}\right)$ for all positive integers $m$, where $N$ is the number of unknowns of the surface density, and $M$ is the number of integration points. We demonstrated the high-order convergence, stability, and accuracy of our algorithm even for extremely small grazing angles, very high frequencies, and surfaces whose height is as many as 40 times its period. In all cases very high accuracies have resulted. The strength of

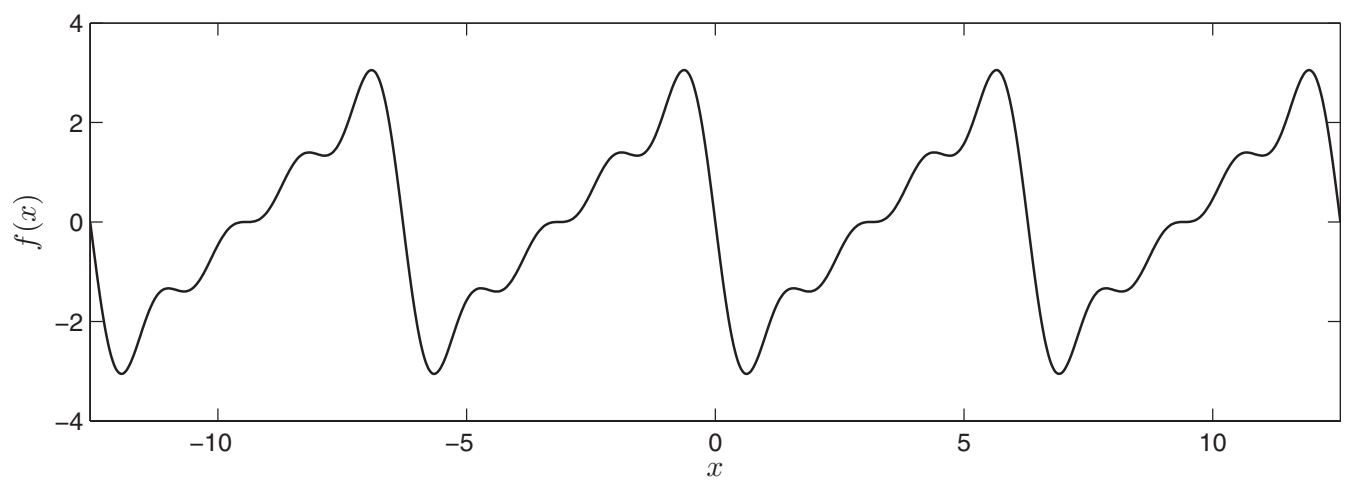

Fig. 2. Composite surface profile Eq. (50): four periods. 
the present method derives from its combined use of Floquet expansions, representation of the kernel functions $K_{1}$ and $K_{2}$ as Chebyshev series, and a high-order integration rule based on integration weights that are obtained accurately by means of asymptotic analysis.

\section{APPENDIX A: PERIODIC FREE-SPACE GREEN'S FUNCTION}

The free-space periodic Green's function $G$ possesses an infinite number of logarithmic singularities located at $x$ $-x^{\prime}=m P, m$ integer; this is explicitly seen from its spatial representation [17]

$$
G\left(\mathbf{r}, \mathbf{r}^{\prime}\right)=\frac{i}{4} \sum_{m=-\infty}^{\infty} e^{i \alpha m P} H_{0}^{(1)}\left(k \sqrt{\left(x-x^{\prime}-m P\right)^{2}+\left(z-z^{\prime}\right)^{2}}\right)
$$

The methodology described in this work requires evaluation of the normal derivative of $G$ over a single period of the interface. The explicit selection of the limits of integration in Eq. (10) as $x^{\prime} \in[x-P / 2, x+P / 2]$ requires that we resolve the singularity at $x=x^{\prime}$ and produce values of the kernel (by evaluating the gradient of $G$ ). From Eq. (A1) it may be seen that in the region $\left|x-x^{\prime}\right|<P$ (i.e., in the region between two logarithmic singularities), the free-space periodic Green's function can be expressed in the form

$$
G\left(\mathbf{r}, \mathbf{r}^{\prime}\right)=\frac{i}{4} H_{0}^{(1)}\left(k\left|\mathbf{r}-\mathbf{r}^{\prime}\right|\right)+\mathcal{D}\left(\mathbf{r}, \mathbf{r}^{\prime}\right)
$$

where $\mathcal{D}$ is an analytic function of its arguments. The form Eq. (A2), of $G$ is particularly useful since it allows us to extract all the kernel singularities; see Section 3.

The efficient numerical evaluation of the free-space periodic Green's function (and its gradient) is an important factor in determining the performance of our algorithm. Inspection of the asymptotic form of the Hankel function ([10], Eq. 9.2.3) indicates that the series, Eq. (A1), exhibits extremely slow convergence: of the order of $\mathcal{O}\left(\mathrm{m}^{-1 / 2}\right)$ as $m \rightarrow \infty$. The spectral series of the Green's function, Eq. (8), on the other hand converges very rapidly away from the singular point $x=x^{\prime}$, but its convergence is prohibitively slow in the neighborhood of $x=x^{\prime}$.

Various techniques for acceleration of convergence of the Green function series are discussed in $[18,19]$. The method introduced by Veysoglu et al. [17] has been especially successful for evaluation of Green's functions for one-dimensional gratings in a small neighborhood of the singularity: it is based on Laplace transform identities that are used to convert the infinite sum in Eq. (A1) into an improper integral whose integrand exhibits exponential decay. Specifically, the algorithm [17] produces the function $\mathcal{D}$ in the form $\mathcal{D}\left(\mathbf{r}, \mathbf{r}^{\prime}\right)=\mathcal{D}_{+}\left(\mathbf{r}, \mathbf{r}^{\prime}\right)+\mathcal{D}_{-}\left(\mathbf{r}, \mathbf{r}^{\prime}\right)$, where

$$
\begin{aligned}
\mathcal{D}_{ \pm}\left(\mathbf{r}, \mathbf{r}^{\prime}\right)= & \frac{1}{\pi} e^{\mp i k\left(x-x^{\prime}\right)} e^{i(k \pm \alpha) P} \int_{0}^{\infty} \mathrm{d} q \\
& \times\left\{\frac{e^{-q^{2} k P \pm q^{2} k\left(x-x^{\prime}\right)}}{1-e^{-q^{2} k P+i(k \pm \alpha) P}} \frac{\cos \left[k\left(z-z^{\prime}\right) q \sqrt{q^{2}-2 i}\right]}{\sqrt{q^{2}-2 i}}\right\} .
\end{aligned}
$$

The exponential decay of the integrands in Eq. (A3) can be exploited to construct, via rather straightforward integration, an efficient algorithm for evaluation of the periodic Green's function $G$; the details of our own implementation are given below.

In a procedure similar to that of DeSanto et al. [3], we evaluate the gradient of the Green's function numerically by means of a combination of two different methods. Selecting a certain value $\delta>0$, for $k\left(z-z^{\prime}\right)>\delta$ we evaluate $x$ and $z$ derivatives of $G$ by direct termwise differentiation of the spectral series [Eq. (8)], and corresponding derivatives of the Hankel function in Eq. (A2) subtracted out to produce the vector function $\nabla \mathcal{D}$. For $k\left(z-z^{\prime}\right) \leqslant \delta$, in turn, explicit spatial derivatives of the integrals, Eq. (A3), are computed numerically as outlined below. Numerical experiments have led us to select the value $\delta=1 / 2$ for the splitting parameter.

We have found it computationally advantageous to evaluate the Veysoglu integrals (and their gradient) by partitioning the infinite domain of integration into a region near 0 and its complement: $[0, \infty]=[0, \varepsilon] \cup[\varepsilon, \infty]$. In the finite subdomain $[0, \varepsilon]$, the integrals are evaluated using FFT-based Clenshaw-Curtis integration [20], while in the infinite interval $[\varepsilon, \infty]$ the integrals are computed using high-order Gauss-Laguerre quadrature ([10], Eq. 25.4.45). Numerical experiments have led us to select the value $\varepsilon=3$, which is used for all results presented in this work. To maintain accuracy, the number $M_{v}$ of terms used for the fast cosine transform required to resolve the derivatives of the Veysoglu integrand for $q \in[0, \varepsilon]$ must be increased with the wavenumber $k$; we select values of $M_{v}$ on the basis of convergence experiments performed for

Table 9. Comparison of the Spatial Collocation and Spectral Testing Methods, in a TM Problem with Scattering Surface Defined in Eq. (49) with $a=P, \lambda=0.1 P, \theta=\pi / 4$ (TE Results Are Nearly Identical) $^{a}$

\begin{tabular}{ccccc}
\hline & \multicolumn{2}{c}{ Spatial Collocation } & \multicolumn{2}{c}{ Spectral Testing } \\
\cline { 2 - 5 }$N$ & $|1-E|$ & Error & $|1-E|$ & Error \\
\hline 60 & $1.67 \times 10^{-1}$ & $6.66 \times 10^{-2}$ & $1.26 \times 10^{-2}$ & $9.56 \times 10^{-3}$ \\
65 & $1.39 \times 10^{-2}$ & $1.03 \times 10^{-2}$ & $1.26 \times 10^{-4}$ & $3.14 \times 10^{-3}$ \\
70 & $1.43 \times 10^{-4}$ & $1.78 \times 10^{-4}$ & $8.59 \times 10^{-5}$ & $1.45 \times 10^{-4}$ \\
75 & $1.80 \times 10^{-6}$ & $1.36 \times 10^{-5}$ & $1.32 \times 10^{-6}$ & $1.28 \times 10^{-6}$ \\
80 & $1.65 \times 10^{-8}$ & $6.47 \times 10^{-8}$ & $2.55 \times 10^{-9}$ & $2.35 \times 10^{-9}$ \\
85 & $7.18 \times 10^{-11}$ & $1.31 \times 10^{-10}$ & $9.10 \times 10^{-13}$ & $6.75 \times 10^{-13}$ \\
90 & $4.00 \times 10^{-14}$ & $3.59 \times 10^{-14}$ & $2.90 \times 10^{-14}$ & $1.60 \times 10^{-14}$ \\
95 & $4.00 \times 10^{-14}$ & $1.70 \times 10^{-14}$ & $1.90 \times 10^{-14}$ & $1.20 \times 10^{-14}$ \\
\hline
\end{tabular}

${ }^{a}$ Parameters: $M=513, M_{v}=257$. The reference solution in each case was obtained by using $N^{*}=100$. Similar performance improvements were observed in a wide range of cases, and no cases were found in which spatial collocation resulted in better performance than its spectral counterpart. 
each problem; the values of $M_{v}$ we used in our experiments are reported in the corresponding table captions. For problems in the classical resonance regime presented in Tables 4 and 8, for example, we used $M_{v}=129$ and 257, respectively, while for the more computationally challenging problems presented in Tables 5-7 we used $M_{v}=1025$. In the grazing-incidence case with $\theta=0.01^{\circ}$ (Table 6) we used $M_{v}=4097$. [As can be seen from Eq. (A3), when $k \pm \alpha$ is small, the integrand is nearly singular in the neighborhood of $q=0$ and, thus, additional sampling is required.] In each case considered in this work, the $[\varepsilon, \infty]$ tails of the Veysoglu integrals were evaluated by means of GaussLaguerre integration using a total of 15 integration points.

\section{APPENDIX B: SPECTRAL TESTING VERSUS SPATIAL COLLOCATION}

As discussed in Subsection 4.A, we have noted that a method based on spectral collocation systematically produces slightly higher accuracies than the spatial collocation approach. This is illustrated in Table 9.

\section{ACKNOWLEDGMENTS}

O. P. Bruno gratefully acknowledges support by the U.S. Air Force Office of Scientific Research (AFOSR) and the National Science Foundation (NSF). M. Haslam gratefully acknowledges the support of the Natural Sciences and Engineering Research Council of Canada.

\section{REFERENCES}

1. R. Petit, ed., Electromagnetic Theory of Gratings, Vol. 22 of Topics in Current Physics (Springer-Verlag, 1980).

2. T. Arens, S. N. Chandler-Wilde, and J. A. DeSanto, "On integral equation and least squares methods for scattering by diffraction gratings," Comm. Comp. Phys. 1, 1010-1042 (2006).

3. J. DeSanto, G. Erdmann, W. Hereman, and M. Misra, "Theoretical and computational aspects of scattering from rough surfaces: one-dimensional perfectly reflecting surfaces," Waves Random Media 8, 385-414 (1998).

4. D. Maystre, "Rigorous vector theories of diffraction gratings," in Progress in Optics, E. Wolf, ed. (NorthHolland, 1984), Vol. XXI.

5. A. G. Voronovich, Wave Scattering From Rough Surfaces (Springer-Verlag, 1994).

6. K. F. Warnick and W. C. Chew, "Numerical simulation methods for rough surface scattering," Waves Random Media 11, R1-R30 (2001).

7. D. Colton and R. Kress, Integral Equation Methods in Scattering Theory (Wiley-Interscience, 1983).

8. D. Colton and R. Kress, Inverse Acoustic and Electromagnetic Scattering Theory, Vol. 93 of Applied Mathematical Sciences, 2nd ed. (Springer-Verlag, 1998).

9. O. P. Bruno and F. Reitich, "Solution of a boundary value problem for the Helmholtz equation via variation of the boundary into the complex domain," Proc. R. Soc. Edinburgh, Sect. A: Math. 122, 317-340 (1992).

10. M. Abramowitz and I. A. Stegun, ed., Handbook of Mathematical Functions With Formulas, Graphs, and Mathematical Tables (Dover, 1992).

11. J. C. Mason and D. C. Handscomb, Chebyshev Polynomials (Chapman \& Hall/CRC, 2003).

12. T. J. Rivlin, Chebyshev Polynomials: From Approximation Theory to Algebra and Number Theory, 2nd ed. (Wiley, 1990).

13. I. S. Gradshteyn and I. M. Ryzhik, Table of Integrals, Series, and Products, 6th ed., Translated from Russian, edited by A. Jeffrey and D. Zwillinger (Academic, 2000).

14. http://www.netlib.org/slatec/.

15. www.netlib.org/lapack/.

16. A. Meier, T. Arens, S. N. Chandler-Wilde, and A. Kirsch, "A Nyström method for a class of integral equations on the real line with applications to scattering by diffraction gratings and rough surfaces," J. Integral Equ. Appl. 12, 281-321 (2000).

17. M. E. Veysoglu, H. A. Yueh, R. T. Shin, and J. A. Kong, "Polarimetric passive remote sensing of periodic surfaces," J. Electromagn. Waves Appl. 5, 267-280 (1991).

18. R. E. Jorgenson and R. Mittra, "Efficient calculation of the free-space periodic Green's function," IEEE Trans. Antennas Propag. 38, 633-642 (1990).

19. A. W. Mathis and A. F. Peterson, "A comparison of acceleration procedures for the two-dimensional periodic Green's function," IEEE Trans. Antennas Propag. 44, 567-571 (1996).

20. W. H. Press, S. A. Teukolsky, W. T. Vetterling, and B. P. Flannery, Numerical Recipes in Fortran 90: The Art of Scientific Computing, 2nd ed. (Cambridge U. Press, 1996). 